\title{
LAS CIENCIAS SOCIALES FRENTE A LA CATEGORÍA DE CLASE SOCIAL
}

\section{SOCIAL SCIENCE VERSUS SOCIAL CLASS CATEGORY Victoria Eugenia González Cárdenas*}

\begin{abstract}
RESUMEN
Este artículo pretende establecer una continuidad entre los inicios de la teoría marxista en la sociedad capitalista europea del siglo xix hasta el posmarxismo de la sociedad capitalista contemporánea, teniendo como hilo conductor, la categoría de clase social en el pensamiento sociológico dentro de su larga trayectoria en el desarrollo histórico social de las ciencias humanas y sociales, en general y de la Sociología, en particular. Asimismo, presenta el análisis de la clase social en la investigación de la desigualdad económica planteado por el marxismo analítico y los aportes de Eric Olín Wright, uno de sus máximos representantes, con la idea de las posiciones contradictorias en las relaciones de clase y la propuesta del concepto de estructura de clases a nivel micro de análisis para la investigación empírica.
\end{abstract}

PALABRAS CLAVE: SOCIOLOGÍA * MARXISMO * CLASE SOCIAL * ESTRUCTURA SOCIAL * INVESTIGACIÓN EMPÍRICA

\section{ABSTRACT}

This article seeks to establish continuity between the beginning of the Marxist theory of capitalist society in European nineteenth century to the post-Marxism of contemporary capitalist society, having as theme the category of social class in sociological thought within his long career in the social historical development of human and social sciences, in general and sociology, in particular. It also presents the analysis of social class in the investigation of economic inequality raised by the analytical Marxism and the contributions of Eric Olin Wright, one of its leading representatives, with the idea of contradictory positions on class relations and the proposal the concept of class structure at the micro level of analysis for empirical research.

KEYWORDS: SOCIOLOGY * MARXISM * SOCIAL CLASS * SOCIAL STRUCTURE * EMPIRICAL RESEARCH

Universidad de Antioquia, Colombia.

vegonzalez@quimbaya.udea.edu.co 


\section{INTRODUCCIÓN}

La categoría de clase social tradicionalmente ligada a la teoría marxista, surge con el pensamiento social y económico en la sociedad capitalista de la Europa del siglo xix. Su análisis se apoya en las disciplinas que conforman las ciencias humanas y sociales, específicamente, la Economía y la Sociología, desde la época de la ilustración hasta nuestros días. Esta categoría tiene una larga trayectoria en el pensamiento sociológico, inscribiéndose en las dos perspectivas teóricas originarias, la liberal y la crítica, las cuales influyeron abiertamente en el análisis de la estratificación social en la sociología académica y la sociología marxista, respectivamente.

La vigencia de la categoría de clase social para el análisis marxista de la sociedad capitalista se haya plenamente justificada, toda vez que siguen existiendo las relaciones de explotación económica y dominación político ideológica, condiciones de desigualdad que muestran la pertinencia teórica y empírica del estudio de la desigualdad, en términos de clases sociales, un concepto que no ha sido superado en el campo de la teoría social.

El objetivo de este artículo es exponer el recorrido histórico social que ha tenido la categoría de clase social desde las ciencias sociales, partiendo desde el siglo xix hasta el xxI, en un intento por responder a la pregunta ¿qué dicen hoy las ciencias sociales frente a la categoría de clase social?

Inicialmente, se exponen los antecedentes de la categoría de clase social desde el desarrollo de la Sociología, para luego analizar la vigencia de la teoría marxista en la Sociología contemporánea y posteriormente, algunas de las tendencias que en la actualidad marcan el análisis marxista de la clase social. Finalmente, a partir del marxismo analítico, se presentan los aportes del sociólogo estadounidense Olín Wright, sobre la idea de las posiciones contradictorias en las relaciones de clase y la propuesta sobre el concepto de estructura de clases a nivel micro de análisis para la investigación empírica.
ANTECEDENTES DE LA CATEGORÍA DE CLASE SOCIAL DESDE LA SOCIOLOGÍA

La categoría de clase social surge con el pensamiento social y económico de los primeros sociólogos que emergieron en el contexto de una sociedad capitalista, la Europa del siglo xix. Por ello, el análisis de la clase social se apoya en la Economía y la Sociología, disciplinas que forman parte de las denominadas ciencias sociales, las cuales a su vez, se inscribieron en el pensamiento intelectual $y$ en el contexto social característico de la época, lo cual influyó abiertamente en el surgimiento $y$ desarrollo de la teoría sociológica y en el análisis de la división social en clases. Como lo expresa Giddens: "la coincidencia del clima político de la Revolución francesa $y$ de los cambios económicos forjados por la Revolución industrial, ofreció el contexto a partir del cual surgió la sociología" (Giddens, 1998: 16).

Las revoluciones políticas que se produjeron a lo largo del siglo xix, desencadenaron el interés de la teoría sociológica por el orden social, en respuesta a la preocupación de algunos teóricos clásicos como Comte y Durkheim por el caos y el desorden social resultante, principalmente en Francia. Asimismo, el capitalismo naciente a partir de los cambios acaecidos por la revolución industrial, produjeron en teóricos clásicos alemanes como Marx y Weber, el interés por el análisis de la división social en clases o estratos, respectivamente. Tanto Marx como Weber fueron críticos severos de la sociedad moderna capitalista, aunque influenciados por pensamientos políticos diferentes que marcaron la división de la Sociología en dos corrientes clásicas, la Sociología marxista y la Sociología académica (Ritzer, 1996).

El antagonismo de estas dos perspectivas teóricas en el contexto de la Europa del siglo xIx, expresa una polémica general en las ciencias sociales, específicamente, en la Ciencia Política y en la Sociología, de la cual se derivó el pensamiento propio de cada corriente. En la Sociología académica predominó el pensamiento liberal, en el cual se concibe la preservación de los derechos individuales frente a las normas impuestas por las colectividades, normas consideradas como limitantes frente 
a dichos derechos. El orden social, preocupación principal de algunos teóricos conservadores de la época como Weber, se supone, es proporcionado por la distribución desigual de los talentos, habilidades e inteligencias que poseen los individuos, orden que solo puede ser preservado por medio de una estructura social que permita la libre competencia y la retribución diferencial de acuerdo a los atributos de cada individuo, puesto que la supresión de las desigualdades sociales iría en contra del logro de un mayor nivel de desarrollo social e individual. Es por ello que las desigualdades, "en tanto expresión de un orden natural preexistente" son consideradas como un elemento inevitable en el desarrollo social a través del tiempo (Mora, Pérez y Cortés, 2004: 12).

En la Sociología marxista, el principio de igualdad propio de esta corriente, hace que se rechace el derecho a la propiedad privada y se abogue por el sometimiento del interés individual al colectivo. El orden social está considerado por la organización del sistema productivo hacia un nuevo sistema, el socialismo, donde cada cual recibe según su trabajo, superando las condiciones de desigualdad ocasionadas por el sistema capitalista como producto del surgimiento de la propiedad privada, enfoque económico que dista del carácter ontológico dado por el pensamiento liberal.

El origen de las desigualdades sociales es atribuido a decisiones sociopolíticas relacionadas con la apropiación y distribución del producto social entre grupos de individuos, dando origen a las clases sociales de acuerdo a la posición que ocupan en el sistema productivo y la relación que guardan con respecto a los medios de producción, a diferencia de la Sociología académica, en la cual la división social está dada por las oportunidades o por los talentos como se mencionó anteriormente. Asimismo, la corriente marxista considera que el sistema capitalista conduce necesariamente a inequidades entre las clases sociales, debido a las relaciones de explotación y dominación que alberga en sí mismo.

La corriente marxista considera la división de la sociedad en clases desde la base económica, en las relaciones de propiedad y no propiedad de los individuos con los medios de producción y en las relaciones de explotación con los productores; en tanto, la Sociología académica, la supone desde las relaciones de poder que se generan en la lucha que se da por el control de las probabilidades de vida y supervivencia entre los individuos implicados, por lo que paralelo a la división en clases se da "la división en estamentos y partidos, según se trate de la distribución del poder económico, social o político" (Duek e Inda, 2006:5). Es por ello que el análisis de la clase social, como forma de abordar la división de la sociedad en clases, se identifica frecuentemente con el marxismo.

Si bien, muchos de los principales elementos del concepto marxista sobre las clases y conflicto entre las mismas se deben al socialista francés Saint-Simón (1760-1825), la teoría general desarrollada por Marx, la superó con creces toda vez que la complementó con los aportes de otras tradiciones teóricas que influyeron de manera decisiva en su pensamiento, como fue la filosofía alemana clásica y la economía política de Adam Smith y David Ricardo (Ríos, 1998). Es característico su enfoque del concepto de clase hacia el funcionamiento de la sociedad, así lo expresa el científico social brasileño Theotonio Dos Santos: "lo que va hacer Karl Marx es exactamente dar al concepto de clase no sólo una dimensión científica sino también atribuirle el papel de base de explicación de la sociedad y de su historia" (Dos Santos, 1976: 12). Sin embargo, a pesar de la importancia que para Marx tenía el concepto de clase, murió justo antes de poder terminar el capítulo de El Capital, dedicado a la conceptualización del mismo. Por consiguiente, las características formales del concepto de clase en Marx tienen que deducirse de una variedad de sus escritos en los que analiza las relaciones de clase en contextos específicos, lo que ha conducido a que diferentes autores marxistas posteriores hayan realizado interpretaciones diferentes sobre las clases sociales en la sociedad capitalista, lo que explica la correspondiente controversia alrededor del tema (García, 2011; Giddens, 1998 y Ritzer, 1993).

Según Marx, la sociedad de clases es el producto de una determinada secuencia de 
cambios históricos que van desde las sociedades primitivas no clasistas, pasando por formas de división del trabajo reducida y propiedad colectiva, hasta llegar propiamente al surgimiento de la sociedad de clases, cuando la expansión de la división del trabajo permite la creación de una producción excedente, la cual queda en manos de unos pocos no productores, dueños de los medios de producción, estableciendo una relación de explotación con los productores carentes de dichos medios (Marx, 1997). De acuerdo con Anthony Giddens, sociólogo inglés, existen dos construcciones conceptuales de la noción de clase que pueden deducirse de los escritos de Marx: "un modelo abstracto o "puro" de dominación de clase que se aplica a todos los sistemas clasistas; $y$ unas descripciones más concretas de las características específicas de las clases en determinadas sociedades" (Giddens, 1973: 29).

El modelo abstracto de Marx, corresponde a un modelo dicotómico que se basa en el argumento sobre la definición de clases por la relación entre los diferentes grupos de individuos con los medios de producción, a condición de una división del trabajo relativamente desarrollada que permita la producción de excedente, dando como resultado dos clases fundamentales en función a las relaciones de propiedad: una minoría de no productores que controla los medios de producción y puede obtener excedente de los productores que son la mayoría. Estas relaciones de propiedad y no propiedad de los agentes con los medios de producción y de explotación con los productores, va definiendo una serie de posiciones que determinan relaciones antagónicas conformando las clases como espacios de relaciones constituidas en oposición unas de otras. Para el marxismo, "las clases no existen por sí mismas sino que se definen por las relaciones que se establecen entre ellas" (Duek e Inda, 2009: 39).

Las clases para Marx se basan en relaciones de mutua dependencia y conflicto, se encuentran en una relación de reciprocidad para mantener su identidad de clase diferenciada $y$ en conflicto por la oposición de intereses derivada de la relación de explotación inherente a la relación de desigualdad, considerada por Marx como una situación injusta y no como una simple diferencia de oportunidades en la vida como lo considera la Sociología académica. Según Marx, esta relación de conflicto que se establece entre las clases puede conducir a la transformación de las estructuras sociales, para lo cual los individuos que conforman una clase deben tomar conciencia de sus intereses comunes, tanto económicos como políticos. Marx destaca el hecho de que "la clase solo se convierte en un agente social importante cuando asume un carácter directamente político, cuando es foco de una acción colectiva. Únicamente bajo ciertas condiciones una clase "en si" se convierte en una clase "para sî" (Giddens, 1973: 32). Es en la lucha, cuando una clase se constituye en sí misma y los intereses que defienden, se convierten en intereses de clase (Marx, 1971).

El determinismo económico de Marx con respecto a las clases sociales, al igual que su pensamiento dialéctico, lo distanciaron de los sociólogos de la corriente académica, que se hallaban influenciados por el pensamiento lineal en términos de causa-efecto heredado de Kant (Wilhelm y Hegel, 1994). Por su parte, Marx, heredó la dialéctica de Hegel, su maestro en Berlín, pero acogió la crítica materialista que Feuerbach le hizo a Hegel y enfocó la dialéctica desde una base material, hacia la relación entre el agente en el nivel micro (el proletario individual) y la estructura macro de la sociedad capitalista (Ritzer, 1993). Marx, manifestó que la raíz de los problemas de la vida moderna podía encontrarse en fuentes materiales como las estructuras del capitalismo y su solución solo podría hallarse en la destrucción de esas estructuras por medio de la acción colectiva de un gran número de personas (Marx y Engels, 1967), lo cual provocó que fuera considerado por los sociólogos conservadores como un revolucionario.

Dentro de la Sociología académica, Weber consideró a diferencia del determinismo económico de Marx, que la base de la estructura social estaba determinada por el poder como elemento constitutivo de las jerarquías en tres dimensiones: económica, social y política. Por ello, consideró la división en clases de acuerdo a la distribución del poder económico 
$y$ la división en estamentos y partidos, según se trate del poder social o político, respectivamente (Duek e Inda, 2006). Este poder fue considerado por Weber como "la probabilidad de imponer la propia voluntad, dentro de una relación social, aun contra toda resistencia $y$ cualquiera que sea el fundamento de esa probabilidad" (Weber, 1964: 43). Definición que expresa en términos individuales, sin considerar los procesos de producción ni las posiciones que pueden ocupar los grupos en dicho proceso, ya que son las cualidades individuales las que según Weber, condicionan las probabilidades de adquirir poder en las diferentes dimensiones, en las cuales se esté compitiendo (Duek e Inda, 2006).

Weber considera que los medios de producción están en el mismo nivel que los dos elementos subjetivos que él incorporó en el análisis de la estratificación social: el estatus, asociado a un determinado grupo social en función de su profesión $y$ al partido, entendido como un grupo de personas que tienen objetivos comunes $y$ trabajan juntas por estos. Al igual que Marx, supone la necesidad de diferenciar entre clase "en sî" y clase "para sí", estableciendo que la relación entre la existencia de intereses de clase similares y el surgimiento de conflicto de clases, está históricamente determinado. A diferencia de la teoría marxista de las clases, Weber con su propuesta tridimensional de la estratificación social, acepta la presencia de grupos sociales paralelos y externos a las clases, es por ello que algunos "teóricos de la estratificación social aceptan la existencia de clases sociales como una subdivisión parcial de una estratificación más integral" (Duek e Inda, 2006: 24).

A diferencia del enfoque de Marx en los factores económicos y sus efectos sobre las ideas, Weber dedicó su atención a las ideas y sus efectos sobre la economía; mientras Marx produjo básicamente una teoría del capitalismo, la obra de Weber constituía una teoría del proceso de racionalización (Brubaker, 1984; Kalberg, 1990 y 2005) que resultó más atractiva para los teóricos posteriores de la Sociología que la teoría marxista, esto se debió en gran parte al carácter polémico de Marx, que lo llevó a ser considerado como alguien que atacaba su propia sociedad. Además, su preocupación centrada exclusivamente en los asuntos referentes a la Economía, permitió que la propuesta tridimensional de la estratificación social de Weber, fuera percibida como más completa. Adicionalmente, la influencia marcada del pensamiento de Kant sobre Weber equivalía a pensar en términos de causa-efecto, lo que era más aceptable para los sociólogos posteriores que no estaban familiarizados con la lógica dialéctica que impulsaba la obra de Marx. Fue así como Weber se convirtió en el sociólogo más importante de su época en Alemania, a diferencia de Marx, cuyas ideas solo adquirieron relevancia a finales de los años 60 (Ritzer, 1993).

\section{VIGENCIA DE LA TEORÍA MARXISTA EN LA SOCIOLOGÍA CONTEMPORÁNEA}

Después de la muerte de Marx en 1883, su colaborador $y$ amigo Engels, fue el primer representante de lo que el sociólogo estadounidense Immanuel Wallerstein denominó la época del "marxismo ortodoxo" (Wallerstein, 1986), caracterizado por un determinismo económico y científico que no tuvo en cuenta la relación dialéctica entre los individuos y las grandes estructuras sociales, al considerar que la teoría científica de Marx "revelaba las leyes económicas que regían el mundo capitalista, estas leyes apuntaban hacia el colapso inevitable del sistema capitalista" (Ritzer, 1993: 51). Este planteamiento parecía no necesitar de la acción, en especial de los trabajadores, excluyendo la acción política, eje fundamental de la postura de Marx, lo cual suscitó la reacción entre los teóricos marxistas que asumían la importancia del individuo, la conciencia, así como la relación entre el pensamiento y la acción.

Es así como surge el marxismo hegeliano que señala la importancia práctica de la acción individual, para inducir la revolución social en aras de acabar con las desigualdades sociales que generan la división en clases, producto del sistema capitalista (Ritzer, 1993: 5). El principal exponente de este punto de vista fue Georg Lukács considerado "el padre fundador del marxismo occidental" (Fischer, 1984). $\mathrm{Su}$ integración del marxismo y la Sociología, 
fue ampliamente desarrollada en los años 20 y 30 con el surgimiento de la teoría crítica como producto de un grupo de neomarxistas alemanes, entre los que se destacan Max Horkheimer, Theodor Adorno, Erich Fromm, Herbert Marcuse y más recientemente, Jurgen Habermas (Ritzer, 1993); quienes se inspiraron en el marxismo hegeliano insatisfechos con la tendencia hacia el determinismo económico al que había llegado la teoría marxista.

En 1923, los teóricos críticos fundaron la escuela crítica de Frankfurt (Jay, 1984), sus estudios se enfocaron en el análisis del sistema cultural basado en la obra de Weber (Greisman, Rietzer $y$ Weber, 1981) empleando rigurosas técnicas científico sociales desarrolladas por los sociólogos americanos. Además, integraron la teoría freudiana centrada en el individuo con los principios sociales $y$ culturales de Marx $y$ Weber, esto les permitió algunas orientaciones distintivas y les dio legitimidad (Ritzer, 1993). El Instituto funcionó en Alemania hasta 1934, luego tuvieron que marcharse a la Universidad de Columbia en New York, debido al hostigamiento del régimen nazi a algunos de sus integrantes por su origen judío.

Una vez finalizó la guerra, tras las presiones para su regreso a Alemania, Horkheimer, el director del Instituto, regresó en 1949, en ese entonces predominaba el marxismo estructural que consideraba el determinismo económico como punto de partida para el análisis de estructuras como el Estado y la ideología con autonomía relativa frente a la Economía, destacándose como principales exponentes, Althusser y Poulantzas. Muchos de los integrantes de la corriente crítica permanecieron en los Estados Unidos, aunque esta corriente solo fue relevante a finales de los años 60 , ya que en esa época la teoría sociológica estadounidense se caracterizó por la influencia del pensamiento liberal. Un estudio realizado sobre la orientación política de la primera teoría sociológica estadounidense (Schwendinger y Schwendinger, 1974), constata que los primeros sociólogos en Estados Unidos fueron políticamente liberales y no conservadores, como la mayoría de los primeros sociólogos europeos; además, existía una escasa crítica del sistema en su conjunto, lo que significa que apenas se cuestionaba el capitalismo.

A finales de los años 60 comenzó a surgir significativamente la teoría marxista en la teoría sociológica estadounidense, cuando a las numerosas críticas realizadas al funcionalismo estructural, teoría sociológica dominante de la época, se sumaron los esfuerzos realizados por la Sociología de Charles Wright Mills y la Teoría del Conflicto, que aunque no representaron una teoría marxista elaborada, sentaron las bases para el desarrollo de una teoría estadounidense arraigada en la tradición marxista. Además, las numerosas protestas que se dieron en los años 60 como la protesta negra, el renacimiento del movimiento feminista, el movimiento estudiantil y la protesta contra la guerra de Vietnam, generaron en los sociólogos jóvenes, atracción por las ideas radicales del pensamiento crítico (Ritzer, 1993). Así es como comenzó a desarrollarse la Sociología marxista en Estados Unidos, a partir de numerosas obras como "La sociología de Marx" de Henri Lefebvre en 1968, cuyo argumento principal: "aunque Marx no era un sociólogo, había mucha sociología en su teoría" (Ritzer, 1993: 89), influyó no solo en el trabajo orientado hacia el marxismo de muchos sociólogos estadounidenses, sino en el apoyo institucional, a través de revistas como Theory and Society, Telos y Marxist Studies.

Los cambios sociales que llevaron a los marxistas a reconsiderar y reconstruir sus teorías, tienen que ver con el fracaso del comunismo a escala mundial (Burawoy, 1989), los cambios mundiales en consonancia con las nuevas corrientes teóricas como el posestructuralismo y el posmodernismo que comparten el interés por la totalidad social, el descentramiento del sujeto, el estudio de las diferencias más que la búsqueda de la unidad; influyeron profundamente en la teoría neomarxista. A esto se suma el surgimiento de un movimiento conocido como marxismo analítico, que se basó en "la necesidad de que las teorías marxistas emplearan los mismos métodos que utilizaba toda empresa científica. Esto condujo a una reinterpretación de Marx en términos intelectuales más convencionales" (Ritzer, 1993: 571), que traza un nuevo horizonte de tendencias para el 
análisis marxista de la clase social en la sociedad contemporánea, una sociedad capitalista en la cual, sigue existiendo aun las relaciones de explotación económica y dominación político ideológica, condiciones de desigualdad que hacen necesario el análisis en términos de clases sociales, un concepto que no ha sido superado en el campo de la teoría social (Duek e Inda, 2009), razón por la cual el análisis en términos de clases sociales, conserva plena vigencia.

\section{TENDENCIAS PARA EL ANÁLISIS MARXISTA DE CLASE SOCIAL}

Las tendencias para el análisis de las clases sociales en la actualidad se circunscriben en las tres grandes corrientes sociológicas: 1) el estructuralismo marxista, enfocado en el análisis de clase desde las grandes estructuras de la sociedad (económica, política e ideológica), a partir de Nicolas Poulantzas; 2) el humanismo teórico que otorga primacía a la conciencia en su definición de las clases, destacándose el concepto de conciencia de clase expuesto por Georg Lukács; y por último, 3) el marxismo analítico, corriente crítica que tiene especial interés en la reformulación de las categorías marxistas, a partir de los datos obtenidos por la investigación empírica. Sus principales exponente son: Gerald Cohen, John Roemer, Jon Elster, Erik Olin Wright, entre otros, siendo Wright quien ha relacionado en sus trabajos la categoría de clase social con el problema de la desigualdad económica (Wright, 2010) y además ha intentado construir dentro de un marco teórico marxista en sentido amplio, un concepto de estructura de clases susceptible de ser usado en el análisis micro a un nivel relativamente bajo de abstracción (Wright, 1992).

El surgimiento del marxismo analítico entre los científicos sociales de habla inglesa durante la década de 1980, constituye según el investigador inglés Perry Anderson (Anderson, 1988), la prueba más contundente de que el centro de la teoría marxista se ha distanciado de Alemania y especialmente, de Francia e Italia. El marxismo analítico, se centra específicamente en asuntos marxistas, los cuales "son abordados utilizando herramientas contemporáneas de la lógica, la matemática y la construcción de modelos" (Roemer, 1986b: 1), adoptando un enfoque no dogmático sobre la teoría de Marx (Gargarella, 1995), la cual es considerada como una forma de ciencia social decimonónica poderosa $y$ válida pero con sus debilidades. Por ello, asume que la vigencia de la teoría de Marx siendo legítima y justificada, requiere la utilización de métodos y técnicas que han surgido a finales del siglo xx. Este enfoque niega que exista una forma de análisis específicamente, marxista o compromiso con un método específico de análisis diferente a los que caracterizan en general a las ciencias sociales (Elster, 1986).

Uno de los principales representantes del marxismo analítico es el sociólogo estadounidense Erik Olin Wright, actualmente presidente de la Asociación Americana de Sociología, cuya contribución está relacionada con su revisión de la teoría marxista de las clases sociales, así como, su esfuerzo por llevar esta revisión teórica al campo de la investigación empírica. Wright considera que adicional a los bienes de capital considerados por Marx, se puede hablar en las sociedades de capitalismo avanzado de dos tipos adicionales de bienes, los de organización que se refieren a la posición jerárquica en la empresa y los de cualificación que hacen referencia a las credenciales educativas. Ambos bienes permiten a los que los poseen explotar al resto de la fuerza de trabajo, pero al mismo tiempo, sus propietarios son explotados por quienes detentan los bienes de capital. Wright sostiene que "el análisis de clase puede funcionar no simplemente como una teoría científica de intereses $y$ conflictos sino también como una teoría emancipatoria de alternativas y de justicia social" (Wright, 1985: 30).

La idea de "las posiciones contradictorias en las relaciones de clase", es una de las principales contribuciones de Wright para el análisis profundo de las clases sociales (Wright, 1985 y 2010), en ella una determinada posición no necesariamente debe estar en una determinada clase social, puede estar simultáneamente en más de una clase. Es por ello que para resolver esta situación contradictoria de clase o el problema de las clases medias, Wright incorporó para clasificar a las personas dos 
dimensiones, autoridad (posición jerárquica) y educación (credenciales educativas), que permiten un abanico de posibilidades para el estudio de las sociedades contemporáneas. Así, intenta resolver situaciones como el problema empírico de donde situar a un gerente que no posee los medios de producción, pero que no tiene la misma situación laboral del proletariado de una fábrica o el problema de un microempresario que es dueño de los medios de producción, pero que no tiene la misma situación que un gran empresario (Rivadulla y Garavito, 2010).

Wright considera que si bien, en la última década se ha progresado en la conceptualización de las clases sociales, no se ha logrado elaborar un concepto de estructura de clases que sea a la vez teóricamente coherente y empíricamente comprehensivo, es por ello que intenta construir dentro de un marco teórico marxista un concepto de estructura de clases susceptible de ser usado en el análisis micro a un nivel relativamente bajo de abstracción (Wright, 1992).

\section{CONCEPTO DE ESTRUCTURA DE CLASES A NIVEL MICRO DE ANÁLISIS}

El análisis de la clase social en la investigación de los diferentes tipos de desigualdades desde las ciencias sociales se ha enfocado a partir de los ingresos económicos; los sociólogos se interesan en el bienestar material porque consideran que la desigualdad que se da en este campo tiene consecuencias para otros fenómenos sociales. Sin embargo, el interés de Wright consiste en elaborar un concepto de estructura de clases que permita el análisis a nivel micro del impacto que las grandes estructuras capitalistas producen sobre la vida de los individuos, se trata de "entender cómo los contextos macroestructurales constriñen los procesos individuales y cómo las elecciones y estrategias de los individuos a nivel micro afectan a los estados macroestructurales" (Wright, 1992: 26).

Para la construcción del concepto estructural de clase a nivel micro, Wright considera necesario explicitar los mecanismos productores de efectos en un plano concreto, que para la tradición marxista se consideran: 1 ) los intereses materiales; 2) la experiencia vivida y 3) las capacidades para la acción colectiva (Wright, 1998). Los intereses materiales tienen que ver tanto con el bienestar económico como con el poder económico y si bien, tanto la clase capitalista como la proletaria tienen el mismo interés por mejorar su bienestar económico, difieren con respecto a los medios para alcanzarlo. Esto se debe a la explotación como concepto base de la apropiación del excedente por parte de los capitalistas, lo que les permite alcanzar un mayor grado de bienestar económico y gozar de niveles mucho más altos de poder económico que los proletarios, quienes al ser explotados, su bienestar económico se resiente y su poder económico se recorta considerablemente, al ser excluidos del control del excedente.

La experiencia vivida hace referencia a las experiencias comunes que comparten un grupo de clase con intereses materiales comunes, como la clase obrera, en la que se presentan tres tipos de experiencias: 1) la experiencia de verse obligado a vender la fuerza de trabajo para sobrevivir; 2) la experiencia de ser dominado $y$ controlado en el trabajo, y 3) la incapacidad para controlar la asignación del excedente social que genera sensación de impotencia frente a las fuerzas sociales. La pérdida del control sobre el propio trabajo y sobre el producto de este es lo que Marx considera como alienación, que al igual que la explotación "nacen de las mismas propiedades relacionales de la producción, pero la explotación se centra principalmente en los intereses materiales y la alienación en las experiencias vitales generadas por aquellas prácticas" (Wright, 1998: 41). En cuanto a las capacidades para la acción colectiva, Wright se refiere a la capacidad de la clase obrera para la lucha colectiva transformadora, que de acuerdo con Marx, se puede dar por dos razones fundamentales: 1) los trabajadores son los productores directos de la riqueza social y poseen colectivamente, el conocimiento necesario para organizar la producción social y 2) la concentración y centralización del capital generada por el capitalismo permite el contacto y la interdependencia de la masa de trabajadores, lo que facilita el contacto para la acción (Wright, 1998). 
Es así como Wright intenta presentar un campo de análisis de la estructura de clase a un nivel micro, a partir de los efectos producidos por las grandes estructuras capitalistas a un nivel macro y que son susceptibles de ser observadas empíricamente por los efectos que ellas producen sobre la vida de los trabajadores, resaltando el análisis de la explotación, la alienación y lucha de clases, en relación con las experiencias vividas en una sociedad capitalista. Cabe anotar que como representante del marxismo analítico, considera la relación dialéctica de dicho proceso, en la cual los efectos producidos a nivel micro tendrán impacto sobre las grandes estructuras macro.

Es importante señalar que Wright considera las clases, no como simples posiciones o roles anclados en la estructura ocupacional, sino que se definen y conforman como tales a partir de las relaciones que se establecen entre sí, en la formación de colectividades, desde la vinculación que desarrollan en el marco de las relaciones sociales de producción. Es por ello, que la estructura de clases no remite a una jerarquía ocupacional o a un esquema gradacional, sino que debe entenderse como un conjunto de posiciones que se definen relacionalmente, representando cada una de ellas un empleo, tratándose, en suma de "un mapa relacional de la estructura de empleos" (Wright, 1992), el cual es afectado por las grandes estructuras capitalistas que producen efectos sobre las personas que conforman clases sociales contrapuestas por la relación y el poder sobre los medios de producción.

\section{CONSIDERACIONES FINALES}

La teoría marxista en las ciencias sociales $y$ humanas, específicamente en el desarrollo de la Sociología desde el siglo xix hasta principios del siglo xxı, ha tenido a través de la categoría de clase social, un hilo conductor que permite el análisis de las desigualdades sociales en la sociedad capitalista, que si bien, ha presentado cambios a través del tiempo, las relaciones de explotación económica y dominación político ideológica consideradas condiciones de desigualdad, aún siguen vigentes.
Dentro del campo de la teoría sociológica, la teoría marxista es ampliamente reconocida como uno de los paradigmas sociológicos más importantes. El determinismo económico que caracterizó la teoría marxista en sus inicios, fue evolucionando hacia una teoría crítica neomarxista que sin desconocer el trabajo de los marxistas económicos, incorporó la actividad humana y los modos en que esa actividad influye en las grandes estructuras sociales.

Las nuevas corrientes teóricas de la Sociología como el posestructuralismo y el posmodernismo que comparten el interés por la totalidad social, el descentramiento del sujeto, el estudio de las diferencias más que la búsqueda de la unidad, han influido profundamente en la teoría neomarxista. A esto se suma el surgimiento del marxismo analítico, que se fundamenta en la necesidad de que las teorías marxistas empleen los métodos científicos para el análisis de los problemas contemporáneos como la desigualdad económica, combinando el análisis teórico riguroso con la investigación empírica, para lo cual Wright propone un análisis de la estructura social a nivel micro, incorporando los conceptos tradicionales expuestos por Marx como la explotación, la alienación y la lucha de clases en contextos contemporáneos dentro de experiencias de vida de los trabajadores, en la realidad capitalista contemporánea.

\section{BIBLIOGRAFÍA}

\section{LIBROS}

Anderson, P. Tras las huellas del materialismo histórico. México: Siglo xxi Editores, 1988.

Brubaker, R. Los Límites de la racionalidad: un ensayo sobre lo social y el pensamiento moral de Max Weber. Londres, Inglaterra: Alle and Unwinns, 1984.

Burawoy, E.M. El consentimiento en la producción: Los cambios del proceso productivo en el capitalismo monopolista. Madrid, España: Ministerio de Trabajo y Seguridad Social, 1989.

Dos Santos, T. Concepto de clases sociales. Buenos Aires, Argentina: Editorial Galerna, 1976. 
Elster, J. "Further thoughts on marxism, functionalism, and game theory". Analytic Marxism. J. E. Roemer (ed.). United States of America. Maison de Sciences de l'homme and Cambridge University Press, 1986: 202-220.

Giddens, A. La estructura de clases en las sociedades avanzadas. Madrid, España: Alianza Editorial, 1973.

Giddens, A. El capitalismo y la moderna teoría social. Barcelona, España: Colección Idea Universitaria, 1998.

Jay, M. La imaginación dialéctica: historia de la escuela de Frankfurt y el instituto de investigación social: 1923-1950. Madrid: Taurus, 1984.

Kalberg, S. "Los tipos de racionalidad de Max Weber: piedras angulares para el análisis del proceso de racionalización de la historia”. Sociedad y religión. Un siglo de controversias en torno a la noción weberiana de racionalización. Aronson PyEWc (ed.). Buenos Aires, Argentina: Prometeo, 2005.

Marx, K. Miseria de la filosofía: respuesta a la filosofía de la miseria del señor Proudhon. Medellín, Colombia: Zeta, 1971.

Marx, K. "El capital". Libro 1. Barcelona, España: Ediciones Curso, 1997.

Marx, K. y Engels, F. La sagrada familia y otros escritos filosóficos de la primera época. Medellín, Colombia: Grijalbo, 1967.

Roemer, J. Analytical marxism. United States of America. Maison de Sciences de l'homme and Cambridge University Press, 1986a: 191-201.

Roemer J. "Introduction". Anatytical Marxism. J. Roemer (ed.). Cambridge, Inglaterra. Cambridge University Press, 1986b: 1-7.

Ritzer, G. Teoría sociológica contemporánea. 3era. Ed. España: McGraw-Hill Interamericana, 1993.

Rivadulla, M.J.A. y Garavito, C.R. (Prólogo a la versión en español). Preguntas a la desigualdad Ensayos sobre análisis de clase, socialismo y marxismo. Erik Olin Wright (ed.). Bogotá, Colombia. Editorial Universidad del Rosario, 2010: 37-42.
Schwendinger, H. y Schwendinger, J.R. The sociologists of the chair: a radical analysis of the formative years of North American sociology (1893-1922). Nueva York, eeuu: Basic Books, 1974.

Weber, M. Economía y sociedad. Esbozo de sociología comprensiva. México: Fondo de Cultura Económica, 1964.

Wilhelm, G. y Hegel, F. Fenomenología del espíritu. México: Fondo de Cultura Económica, 1994.

Wright, O. Classes. Great Britain: Verso Editions, 1985.

Wright, O. "¿Qué tienen las clases en común para ser clases?". Marx y el Siglo XXI. Hacia un marxismo ecológico y crítico del progreso. Renán Vega (ed.). Santafe de Bogotá, Colombia. Ediciones Pensamiento Crítico-Editorial Antropos, 1998: 31-55.

Wright, O. Preguntas a la desigualdad. Ensayos sobre análisis de clase, socialismo y marxismo. Bogotá, Colombia: Editorial Universidad del Rosario, 2010.

\section{REVISTAS}

Duek, C. e Inda, G. "La teoría de la estratificación social de Weber: un análisis crítico". Revista Austral de Ciencias Sociales 11. Chile. Facultad de Filosofía y Humanidades, Instituto de Ciencias Sociales, Universidad Austral, 2006: 5-24.

Duek, C. e Inda, G. "¿Desembarazarse de Marx? Avatares del concepto de clases sociales". Revista Conflicto Social 1. Argentina. Programa de Investigaciones sobre Conflicto Social, Universidad de Buenos Aires, 2009: 26-55.

Fischer, N. "Hegelian Marxism and Ethics". Canadian Journal of Political and Social Theory 8. Canada. Universidad de Concordia,1984: 112-38.

Gargarella, R. "Marxismo analítico, el marxismo claro". Doxa 17-18. España. Universidad de Alicante, 1995: 231-55.

Greisman, H.C.; Ritzer, G. y Weber, M. "Critical Theory and the administered world". Qualitative Sociology 4. Países Bajos. Srpinger, 1981: 34-5. 
Kalberg, S. "The rationalization of action in Max Weber's sociology of religion". Sociological Theory 1 (8). Eeuu. American Sociological Association (ASA), 1990: 58-84.

Mora, M.; Pérez, J. y Cortés, F. "Desigualdad social en América Latina. Viejos problemas, nuevos debates". Cuaderno de Ciencias Sociales 131. Costa Rica. Facultad Latinoamericana de Ciencias Sociales (Flacso), 2004. En: <http:// bibliotecavirtual.clacso.org.ar/Costa Rica/flacso-cr/20120809035816/cuad131. pdf>

Ríos, J. "Las teorías de las clases sociales de Marx y de Weber: Introducción para estudiosos de la administración". Contaduría y Administración 189. México. Facultad de Contaduría y Administración, Universidad Nacional Autónoma de México, 1998: 19-28.

Wallerstein, I. "Marxisms as utopias: evolving ideologies". American Journal of Sociology Published 6 (91). Eeuu.
The University of Chicago Press, 1986: 1295-308.

Wright, O. "Reflexionando, una vez más, sobre el concepto de estructura de clases". Zona abierta 59-60. España. Fundación Pablo Iglesias, 1992: 17-126.

TEXTOS ELECTRÓNICOS

García, M.J. "Teorías marxistas de las clases sociales". [Tesina de grado]. Universidad Nacional de Cuyo, 2011. En: <http:// bdigital.uncu.edu.ar/3705> [consultado el 15 de mayo de 2012].

Marx, C. Carta de Marx a Weydemeyer del 5 de marzo de 1852. Nueva York, 1852. En: <www.ucm.es/info/bas/es/marx-eng/ cartas/oe1/mrxoe118.htm $>$ [consultado el 4 de junio de 2012].

Fecha de ingreso: 03/07/2013 Fecha de aprobación: 16/01/2014 
